\title{
Genome sequence of a distinct watermelon mosaic virus identified from ginseng (Panax ginseng) transcriptome
}

\author{
D. PARK, H. KIM, Y. HAHN
}

Department of Life Science, Research Center for Biomolecules and Biosystems, Chung-Ang University, Seoul 06974, South Korea

Received March 9, 2017; accepted May 19, 20117

\begin{abstract}
Summary. - Watermelon mosaic virus (WMV) is a member of the genus Potyvirus, which is the largest genus of plant viruses. WMV is a significant pathogen of crop plants, including Cucurbitaceae species. A WMV strain, designated as WMV-Pg, was identified in transcriptome data collected from ginseng (Panax ginseng) root. WMV-Pg showed $84 \%$ nucleotide sequence identity and $91 \%$ amino acid sequence identity with its closest related virus, WMV-Fr. A phylogenetic analysis of WMV-Pg with other WMVs and soybean mosaic viruses (SMVs) indicated that WMV-Pg is a distinct subtype of the WMV/SMV group of the genus Potyvirus in the family Potyviridae.
\end{abstract}

Keywords: watermelon mosaic virus; Potyvirus; genome; ginseng; Panax ginseng

\section{Introduction}

Potyvirus, the largest genus of plant viruses, causes considerable crop loss worldwide (Revers and Garcia, 2015). Potyvirus virion is a non-enveloped virus, with filamentous and flexuous capsid, and contains a linear positive-sense singlestranded (ss) RNA genome. The genome encodes an open reading frame (ORF) encoding a polyprotein that is cleaved into 10 mature proteins: P1-pro, HC-pro, P3, 6K1, CI, 6K2, VPg, NIa-pro, NIb-RdRp, and CP. The three virus-encoded proteases, $\mathrm{P} 1$ protease (P1-pro), helper component protease (HC-pro), and nuclear inclusion a protease (NIa-pro), are involved in this processing. P1-pro and HC-pro cleave a site at their own C-terminus, whereas NIa-pro is responsible for the proteolytic, co-translational processing of the remaining seven sites in cis and in trans manner (Adams et al., 2005). In addition, a smaller polyprotein is also produced via a polymerase slippage mechanism within the $\mathrm{P} 3$ region,

*Corresponding author. E-mail: hahny@cau.ac.kr; phone: +82-2820-5812.

Abbreviations: $\mathrm{BCMV}=$ bean common mosaic virus; $\mathrm{CLLV}=$ calla lily latent virus; $\mathrm{EAPV}=$ East Asian passiflora virus; $\mathrm{SMV}=$ soybean mosaic virus; $\mathrm{WMV}=$ watermelon mosaic virus; $\mathrm{WVMV}=$ wisteria vein mosaic virus which is cleaved to P1-pro, HC-pro, and P3N-PIPO. These mature proteins are required for replication, translation, packaging, movement, and transmission of the virus.

Watermelon mosaic virus (WMV) is a member of the genus Potyvirus with a relatively broad host range, and it is a significant pathogen of the Cucurbitaceae family plants (squashes, pumpkins, zucchinis, watermelons, cucumbers, and melons) in temperate and Mediterranean regions (Ali et al., 2006). WMV was also identified in Korean ginseng (Panax ginseng), which was designated as WMV-Insam (Jung et al., 2013). WMV belongs to the bean common mosaic virus (BCMV) subgroup. The sequence identity of $\mathrm{CP}$ and the 3 '-untranslated region (UTR) suggests that WMV and soybean mosaic virus (SMV) are two strains of the same species, although the biological and serological features of WMV and SMV differ (Frenkel et al., 1989). There is evidence suggesting that WMV originated via interspecific recombination event between SMV-like and BCMV-like viruses within the P1 region (Desbiez and Lecoq, 2004). Moreover, multiple intraspecific recombinants among WMV strains have been identified; therefore, classification of these viruses solely based on a portion of the genome is difficult (Desbiez and Lecoq, 2008).

In this study, transcriptome data collected from ginseng in China were analyzed to identify RNA viruses that infect ginseng and a genomic sequence of a distinct WMV strain 
was identified. Sequence and phylogenetic analyses were performed to determine the relationship of this strain among various strains in the BCMV subgroup.

\section{Materials and Methods}

Sequence analysis. Ginseng RNA-seq data (18 separate runs) were downloaded from the Sequence Read Archive (SRA) of the National Center for Biotechnology Information (NCBI; accession number PRJNA264354) (Wu et al., 2015). Complete genome sequences of double-stranded (ds) RNA and ssRNA viruses were downloaded from the NCBI nucleotide database. BLASTN searches were performed to identify RNA virus-associated reads in the ginseng transcriptome data using the known RNA viral genome sequences as queries (Altschul et al., 1997). SPAdes (version 3.7.1) was used to assemble collected virus reads into a contig (Bankevich et al., 2012). Then, a BLASTN search was repeated using the contig as query to collect leftover virus reads and to extend the contig. The UPARSE agglomerative clustering method implemented in USEARCH was used to cluster viral sequences, with an identity threshold of 0.9 (Edgar, 2013). Multiple sequence alignment was performed with MUSCLE, and a phylogenetic tree was inferred using the neighbor-joining method in MEGA7 (Kumar et al., 2016)

\section{Results and Discussion}

To identify novel RNA viruses that infect ginseng, 99.6 gigabases of RNA-seq data (18 separate runs) that were obtained from ginseng roots, stems, and leaves collected in China were analyzed (Wu et al., 2015). A contig of 10,046 bp was assembled from a RNA-seq run derived from ginseng root (SRA accession number: SRR1653717). A sequence similarity search against all known viral sequences showed that the contig was a full-length genome of a novel member of the genus Potyvirus. The closest virus was a WMV-Fr strain with $84 \%$ overall nucleotide sequence identity and $91 \%$ amino acid sequence identity. Because it was a distinct WMV identified from ginseng (Panax ginseng), the putative virus was designated as WMV-Pg.

The WMV-Pg genome (NCBI Acc. No. KX926428) has an ORF encoding a polyprotein of 3,218 amino acids with nine putative cleavage sites, which are predicted to produce 10 mature proteins (Fig. 1 and Supplementary Table S1). The cleavage sites producing P1-pro and HC-pro were concordant with the consensus sequences I-X-H-Y/S and $\mathrm{Y}-\mathrm{X}-\mathrm{V}-\mathrm{G} / \mathrm{G}$, respectively, where $\mathrm{X}$ is any amino acid and "/" is the cleavage site. The other seven sites cleaved by NIa-pro have conserved motifs of $\mathrm{V}-\mathrm{X}-\mathrm{X}-[\mathrm{QE}] /[\mathrm{SAG}]$, which are in agreement with previously reported cleavage sites in other Potyvirus species (Adams et al., 2005). An alternative ORF PIPO generated by polymerase slippage which would produce the $11^{\text {th }}$ mature protein, P3N-PIPO, was identified.

The overall sequence identities between WMV-Pg and representative Potyvirus species of the BCMV subgroup are shown in Table 1 (amino acid) and Supplementary Table S2 (nucleotide). The nucleotide and amino acid sequence identities were $71-84 \%$ and $71-91 \%$, respectively, and the
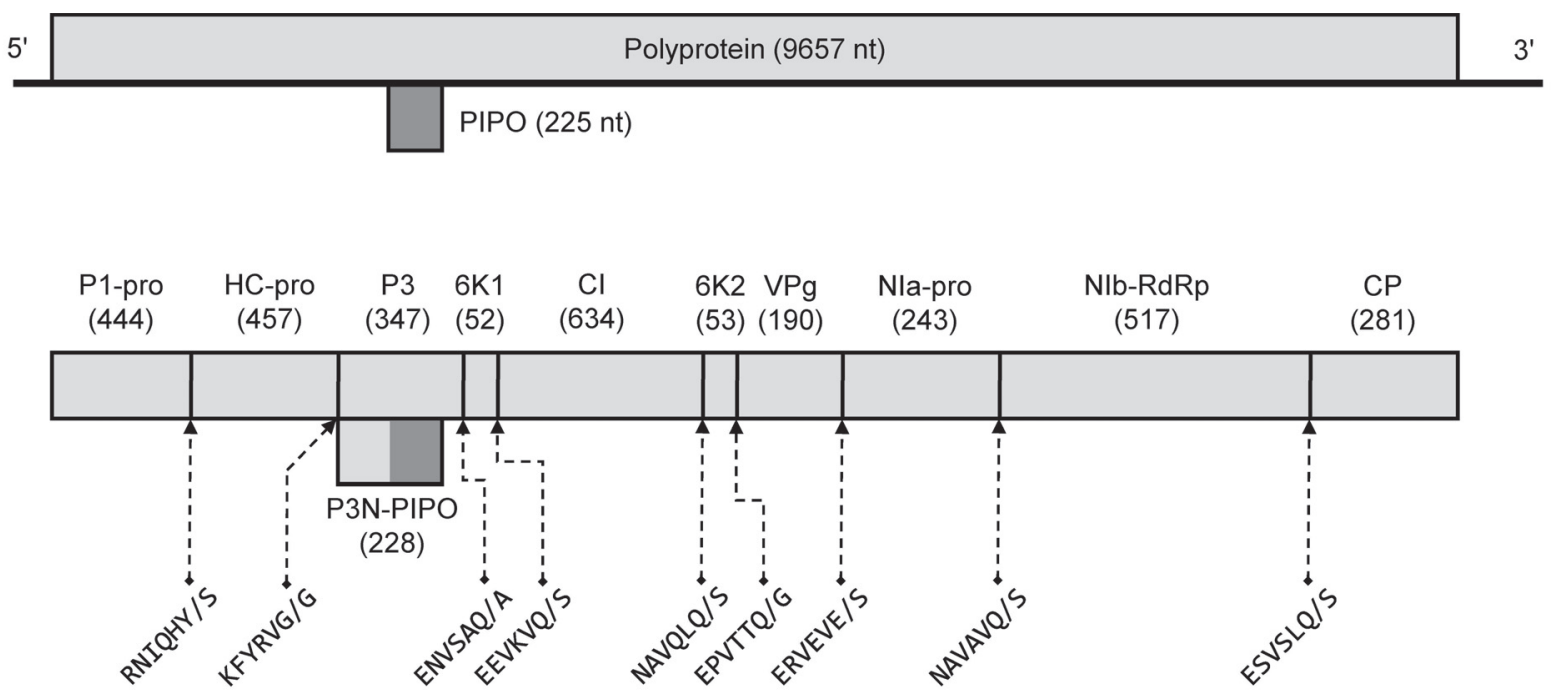

Fig. 1

Genomic structure of WMV-Pg

Open reading frames (top) and mature proteins (bottom) are depicted with their nt and aa sizes shown in parentheses. Predicted proteolytic cleavage sites are presented below. 
Table 1. Amino acid sequence identities (\%) between WMV-Pg and related Potyvirus species

\begin{tabular}{|c|c|c|c|c|c|c|c|c|c|c|c|c|c|}
\hline Virus & Acc. No. & Overall & $\begin{array}{l}\text { Overall, } \\
\text { excluding } \\
\text { P1-pro }\end{array}$ & P1-pro & HC-pro & P3 & $6 \mathrm{~K} 1$ & CI & $6 \mathrm{~K} 2$ & VPg & NIa-pro & NIb-RdRp & $\mathrm{CP}$ \\
\hline WMV-Fr & NC_006262 & 91 & 93 & 77 & 91 & 89 & 100 & 96 & 96 & 91 & 96 & 92 & 90 \\
\hline SMV & NC_002634 & 86 & 90 & $56^{*}$ & 90 & 80 & 92 & 92 & 94 & 89 & 95 & 92 & 84 \\
\hline WVMV & NC_007216 & 81 & 85 & $46^{*}$ & 85 & 72 & 88 & 91 & 83 & 88 & 89 & 86 & 76 \\
\hline CLLV & NC_021196 & 82 & 88 & $25^{*}$ & 88 & 81 & 86 & 92 & 93 & 89 & 92 & 91 & 80 \\
\hline EAPV & NC_007728 & 72 & 77 & $42^{*}$ & 78 & 58 & 77 & 82 & 74 & 76 & 83 & 82 & 74 \\
\hline FVY & NC_010954 & 73 & 76 & $38^{*}$ & 79 & 55 & 77 & 82 & 77 & 73 & 79 & 82 & 75 \\
\hline BCMV & NC_003397 & 71 & 73 & $52^{*}$ & 73 & 50 & 81 & 78 & 62 & 79 & 79 & 79 & 74 \\
\hline BCMNV & NC_004047 & 72 & 75 & $38^{*}$ & 76 & 48 & 85 & 81 & 71 & 78 & 77 & 83 & 73 \\
\hline
\end{tabular}

${ }^{\star}$ Partial alignment.

most closely related viruses were WMV-Fr, SMV, wisteria vein mosaic virus (WVMV), calla lily latent virus (CLLV), East Asian passiflora virus (EAPV), fritillary virus Y (FVY), $\mathrm{BCMV}$, and bean common mosaic necrosis virus (BCMNV). The 5'-UTR of WMV-Pg showed sequence similarity to WMV-Fr and BCMV (see Supplementary Table S2), which supported the previous observation that WMV originated from an ancestral recombination event between a BCMVlike virus and a SMV-like virus that occurred within the P1 region (Desbiez and Lecoq, 2004).

For the phylogenetic analysis of WMV-Pg and related Potyvirus species, the whole genome sequences of WMV, SMV, CLLV, WVMV, and EAPV strains were identified using a BLASTN search of the NCBI database. Collected sequences were clustered using the UPARSE agglomerative clustering method implemented in USEARCH, with an identity threshold of 0.9 (Edgar, 2013). When there were multiple sequences in a cluster, one representative sequence was selected (Supplementary Table S3). A multiple alignment of representative sequences was constructed and a phylogenetic tree was inferred using the neighborjoining method (Fig. 2). In this tree, WMV-Pg did not form a cluster with any other strains, indicating that WMV-Pg is a distinct subtype. All previously reported WMVs, except WMV-KF274031, were clustered into one group represented by WMV-Fr, which is the virus most closely

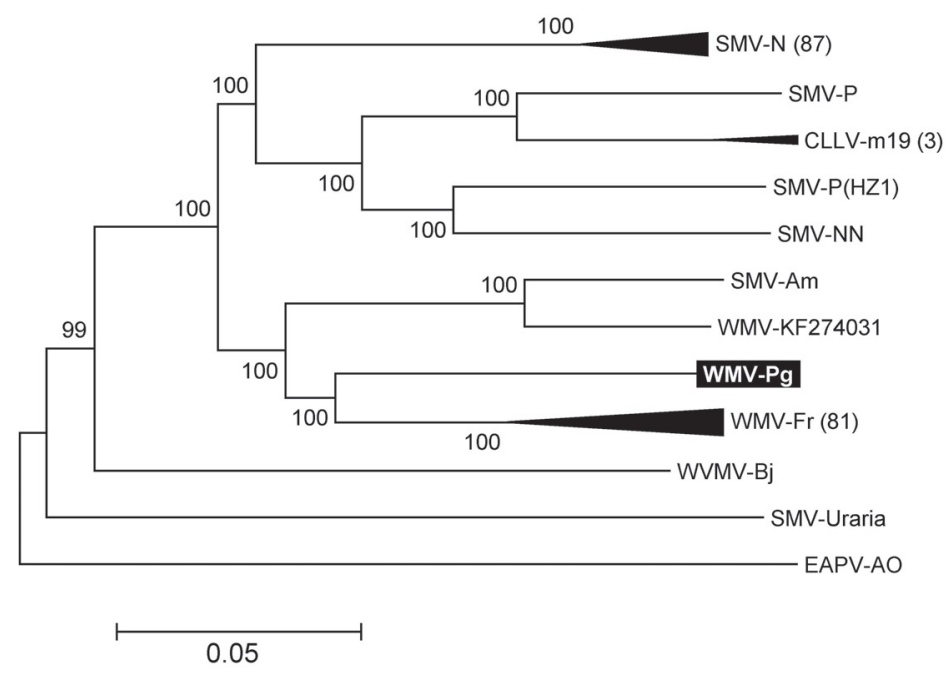

Fig. 2

Phylogenetic tree of WMV-Pg and related representative Potyvirus isolates

The isolates were clustered based on sequence similarity. The SMV-N, CLLV-m19, and WMV-Fr clusters contain 87, 3, and 81 sequences, respectively. Numbers at the nodes are the bootstrap values (percentages) of 1,000 replicates. The EAPV-AO strain was used as an outgroup. See Supplementary Table S3 for the list of sequences analyzed. 
related to WMV-Pg. The WMV-Fr cluster contained 81 strains and isolates collected from various plant species in wide geographic regions. The strains were isolated from various Cucurbitaceae species, Dendrobium anosmum (orchid), and Minthostachys verticillata (peperina) in Europe (France and Italy), America (USA, Argentina, Venezuela, and Chile) and Asia (Turkey, Iran, Pakistan, India, China, and South Korea). Interestingly, there were at least $23 \mathrm{WMV}$ isolates collected from Panax ginseng in South Korea (see Supplementary Table S3). However, these isolates were clustered with WMV-Fr but not with WMV-Pg. Therefore, we conclude that WMV-Pg is a distinct strain that may infect ginseng.

The phylogenetic relationships among WMV/SMVrelated viruses suggest that a taxonomic reorganization of these viruses should be considered. For example, SMV-Am formed a clade with WMV-KF274031, which, in turn, was in a sister clade of the WMV-Pg/WMV-Fr clade; the CLLVm19 cluster was in a clade embedded within the SMVs; and SMV-Uraria was more distant from the WMV/SMV group than WVMV-Bj. Therefore, a systematic regrouping of these viruses is required.

In conclusion, the genome sequence of WMV-Pg was identified in an analysis of Panax ginseng root RNA-seq data. We have showed that, WMV-Pg is a novel distinct subtype of the WMV/SMV group of the genus Potyvirus of the family Potyviridae.

Acknowledgement. This research was supported by the ChungAng University Research Scholarship Grants in 2016 and by the National Research Foundation of Korea funded by the Korea Government [2017R1A1B4005866].

Supplementary information is available in the online version of the paper.

\section{References}

Adams MJ, Antoniw JF, Beaudoin F (2005): Overview and analysis of the polyprotein cleavage sites in the family Potyviridae. Mol. Plant Pathol. 6, 471-487. https://doi.org/10.1111/ j.1364-3703.2005.00296.x

Ali A, Natsuaki T, Okuda S (2006): The complete nucleotide sequence of a Pakistani isolate of Watermelon mosaic virus provides further insights into the taxonomic status in the bean common mosaic virus subgroup. Virus Genes 32, 307-311. https://doi.org/10.1007/s11262-005-6915-Z

Altschul SF, Madden TL, Schaffer AA, Zhang J, Zhang Z, Miller W, Lipman DJ (1997): Gapped BLAST and PSI-BLAST: a new generation of protein database search programs. Nucleic Acids Res. 25, 3389-3402. https://doi.org/10.1093/ nar/25.17.3389

Bankevich A, Nurk S, Antipov D, Gurevich AA, Dvorkin M, Kulikov AS, Lesin VM, Nikolenko SI, Pham S, Prjibelski AD, Pyshkin AV, Sirotkin AV, Vyahhi N, Tesler G, Alekseyev MA, Pevzner PA (2012): SPAdes: a new genome assembly algorithm and its applications to single-cell sequencing. J. Comput. Biol. 19, 455-477. https://doi.org/10.1089/ cmb.2012.0021

Desbiez C, Lecoq H (2004): The nucleotide sequence of watermelon mosaic virus (WMV, Potyvirus) reveals interspecific recombination between two related potyviruses in the $5^{\prime}$ part of the genome. Arch. Virol. 149, 1619-1632. https:// doi.org/10.1007/s00705-004-0340-9

Desbiez C, Lecoq H (2008): Evidence for multiple intraspecific recombinants in natural populations of watermelon mosaic virus (WMV, Potyvirus). Arch. Virol. 153, 1749-1754. https://doi.org/10.1007/s00705-008-0170-2

Edgar RC (2013): UPARSE: highly accurate OTU sequences from microbial amplicon reads. Nat. Methods 10, 996-998. https://doi.org/10.1038/nmeth.2604

Frenkel MJ, Ward CW, Shukla DD (1989): The use of 3' non-coding nucleotide-sequences in the taxonomy of potyviruses: application to watermelon mosaic virus- 2 and soybean mosaic virus-N. J. Gen. Virol. 70, 2775-2783. https://doi. org/10.1099/0022-1317-70-10-2775

Jung W-K, Nam M, Lee JH, Park CY, Kim BH, Park EH, Lee M-A, Kim M-K, Choi H-S, Lee JS, Kim J-S, Choi JK, Kwon TR, Lee K-W, Lee S-H (2013): Novel pathogenic strain of watermelon mosaic virus occurred on Insam (Panax ginseng). Res. Plant Dis. 19, 331-337. https://doi. org/10.5423/RPD.2013.19.4.331

Kumar S, Stecher G, Tamura K (2016): MEGA7: Molecular evolutionary genetics analysis version 7.0 for bigger datasets. Mol. Biol. Evol. 33, 1870-1874. https://doi.org/10.1093/ molbev/msw054

Revers F, Garcia JA (2015): Molecular biology of potyviruses. Adv. Virus Res. 92, 101-199. https://doi.org/10.1016/ bs.aivir.2014.11.006

Wu B, Long Q, Gao Y, Wang Z, Shao T, Liu Y, Li Y, Ding W (2015): Comprehensive characterization of a time-course transcriptional response induced by autotoxins in Panax ginseng using RNA-Seq. BMC Genomics 16, 1010. https:// doi.org/10.1186/s12864-015-2151-7 


\title{
Supplementary information
}

\section{Genome sequence of a distinct watermelon mosaic virus identified from ginseng (Panax ginseng) transcriptome}

\author{
D. PARK, H. KIM, Y. HAHN
}

Department of Life Science, Research Center for Biomolecules and Biosystems, Chung-Ang University, Seoul 06974, South Korea

Received March 9, 2017; accepted May 19, 20117

Supplementary Table S1. Genomic features of WMV-Pg

\begin{tabular}{|c|c|c|c|}
\hline Feature & Mature protein & Genome position & Protein position \\
\hline 5'-UTR & & $1-116$ & \\
\hline \multirow[t]{11}{*}{ polyprotein } & & $117-9773$ & $1-3218$ \\
\hline & P1-pro & $117-1448$ & $1-444$ \\
\hline & HC-pro & $1449-2819$ & $445-901$ \\
\hline & P3 & $2820-3860$ & $902-1248$ \\
\hline & $6 \mathrm{~K} 1$ & $3861-4016$ & $1249-1300$ \\
\hline & $\mathrm{CI}$ & $4017-5918$ & $1301-1934$ \\
\hline & $6 \mathrm{~K} 2$ & $5919-6077$ & 1935-1987 \\
\hline & VPg & $6078-6647$ & $1988-2177$ \\
\hline & NIa-pro & $6648-7376$ & $2178-2420$ \\
\hline & NIb-RdRp & $7377-8927$ & $2421-2937$ \\
\hline & $\mathrm{CP}$ & $8928-9770$ & $2938-3218$ \\
\hline PIPO & & $3278-3502^{*}$ & $1-65^{*}$ \\
\hline 3'-UTR & & 9774-10046 & \\
\hline
\end{tabular}

"Only the PIPO ORF is shown. The mature protein P3N-PIPO is 227 amino acids long.

Supplementary Table S2. Nucleotide sequence identities (\%) between WMV-Pg and related Potyvirus species

\begin{tabular}{llcccccccccccccc}
\hline Virus & Acc. No. & Overall & $\begin{array}{c}\text { Overall } \\
\text { excluding } \\
\text { 5'-UTR } \\
\text { \& P1-pro }\end{array}$ & 5'-UTR & P1-pro & HC-pro & P3 & 6K1 & CI & 6K2 & VPg & NIa-pro & NIb-RdRp & CP & 3'-UTR \\
\hline WMV-Fr & NC_006262 & 84 & 85 & $81^{*}$ & 79 & 84 & 86 & 86 & 85 & 83 & 85 & 86 & 84 & 86 & 88 \\
SMV & NC_002634 & 80 & 81 & - & $75^{*}$ & 81 & 80 & 84 & 82 & 87 & 81 & 82 & 81 & 85 & 77 \\
WVMV & NC_007216 & 77 & 77 & - & $70^{*}$ & 76 & 75 & 84 & 79 & 82 & 79 & 78 & 77 & 81 & 76 \\
CLLV & NC_021196 & 81 & 81 & - & - & 80 & 80 & 82 & 81 & 82 & 82 & 82 & 81 & 85 & 78 \\
EAPV & NC_007728 & 73 & 73 & - & - & 71 & 71 & 78 & 74 & 79 & 74 & 74 & 75 & 76 & - \\
FVY & NC_010954 & 73 & 73 & - & - & 72 & 69 & - & 72 & 78 & 75 & 73 & 75 & 74 & 71 \\
BCMV & NC_003397 & 71 & 71 & $71^{*}$ & $74^{*}$ & 73 & 67 & 75 & 72 & 79 & 77 & 73 & 73 & 75 & 69 \\
BCMNV & NC_004047 & 73 & 73 & - & - & 72 & 67 & 76 & 72 & 83 & 74 & 74 & 74 & 74 & 78 \\
\hline
\end{tabular}

${ }^{\star}$ Partial alignment. 
Supplementary Table S3. List of the Potyvirus species used for the phylogenetic analysis

A. List of the representative viruses

\begin{tabular}{lllllll}
\hline Label & Acc. No. & Species & Strain & Isolate & Host & Country \\
\hline SMV-N & NC_002634 & SMV & N & - & - & - \\
SMV-P & AJ507388 & SMV & P & - & Pinellia ternata & China:Zhejiang \\
CLLV-m19 & NC_021196 & CLLV & m19 & - & Calla lily & Taiwan \\
SMV-P(HZ1) & AJ628750 & SMV & P & HZ1 & Pinellia ternata & China:Zhejiang: Hangzhou \\
SMV-NN & KF982784 & SMV & - & NN & Pinellia pedatisecta & China \\
SMV-Am & KC845322 & SMV & - & Am & Atractylodes macrocephala & China \\
WMV-KF274031 & KF274031 & WMV & - & - & Ailanthus altissima & China \\
WMV-Fr & NC_006262 & WMV & WMV-Fr & - & - & - \\
WVMV-Bj & NC_007216 & WVMV & - & Beijing & Wisteria & China \\
SMV-Uraria & LC037232 & SMV & Uraria & - & Uraria crinita & Taiwan:Pingtung \\
EAPV-AO & NC_007728 & EAPV & AO & AO & - & Japan:Kagoshima, Amami-O-shima \\
\hline
\end{tabular}

B. List of the 87 viruses of the "SMV-N" cluster

\begin{tabular}{|c|c|c|c|c|c|c|}
\hline No. & Acc. No. & Species & Strain & Isolate & Host & Country \\
\hline 1 & $\mathrm{AB} 100442$ & SMV & - & $\mathrm{Aa}$ & - & - \\
\hline 2 & AB100443 & SMV & - & Aa15-M2 & - & - \\
\hline 3 & AF241739 & SMV & G7 & - & - & - \\
\hline 4 & AJ310200 & SMV & Huanghuai strain 5 & SAAS & soybean & China:Shandong \\
\hline 5 & AJ312439 & SMV & severe & Hangzhou & soybean & China:Zhejiang \\
\hline 6 & AJ619757 & SMV & - & CN18 & - & South Korea:Daejeon \\
\hline 7 & AY216010 & SMV & G7 & - & - & - \\
\hline 8 & AY216987 & SMV & G7d & - & - & - \\
\hline 9 & AY294044 & SMV & G5 & - & Glycine max & South Korea \\
\hline 10 & AY294045 & SMV & G7H & - & Glycine max & South Korea \\
\hline 11 & NC_002634 & SMV & $\mathrm{N}$ & - & - & - \\
\hline 12 & EU871724 & SMV & - & $\mathrm{L}$ & Glycine max & Canada \\
\hline 13 & EU871725 & SMV & - & $\mathrm{L}-\mathrm{RB}$ & Glycine max & Canada \\
\hline 14 & FJ376388 & SMV & $\mathrm{G} 5 \mathrm{H}$ & - & Glycine max & South Korea \\
\hline 15 & FJ548849 & SMV & - & WS200 & wild soybean & South Korea \\
\hline 16 & FJ640954 & SMV & - & WS32 & wild soybean & South Korea \\
\hline 17 & FJ640955 & SMV & - & WS37 & wild soybean & South Korea \\
\hline 18 & FJ640956 & SMV & - & WS84 & wild soybean & South Korea \\
\hline 19 & FJ640957 & SMV & - & WS101 & wild soybean & South Korea \\
\hline 20 & FJ640958 & SMV & - & WS105 & wild soybean & South Korea \\
\hline 21 & FJ640959 & SMV & - & WS109 & wild soybean & South Korea \\
\hline 22 & FJ640960 & SMV & - & WS110 & wild soybean & South Korea \\
\hline 23 & FJ640961 & SMV & - & WS116 & wild soybean & South Korea \\
\hline 24 & FJ640962 & SMV & - & WS117 & wild soybean & South Korea \\
\hline 25 & FJ640963 & SMV & - & WS128 & wild soybean & South Korea \\
\hline 26 & FJ640964 & SMV & - & WS132 & wild soybean & South Korea \\
\hline 27 & FJ640965 & SMV & - & WS135 & wild soybean & South Korea \\
\hline 28 & FJ640966 & SMV & - & WS144 & wild soybean & South Korea \\
\hline 29 & FJ640967 & SMV & - & WS145 & wild soybean & South Korea \\
\hline 30 & FJ640968 & SMV & - & WS149 & wild soybean & South Korea \\
\hline 31 & FJ640969 & SMV & - & WS151 & wild soybean & South Korea \\
\hline 32 & FJ640970 & SMV & - & WS155 & wild soybean & South Korea \\
\hline 33 & FJ640971 & SMV & - & WS156 & wild soybean & South Korea \\
\hline 34 & FJ640972 & SMV & - & WS160 & wild soybean & South Korea \\
\hline
\end{tabular}


B. List of the 87 viruses of the "SMV-N" cluster (continued)

\begin{tabular}{|c|c|c|c|c|c|c|}
\hline No. & Acc. No. & Species & Strain & Isolate & Host & Country \\
\hline 35 & FJ640973 & SMV & - & WS162 & wild soybean & South Korea \\
\hline 36 & FJ640974 & SMV & - & WS202 & wild soybean & South Korea \\
\hline 37 & FJ640975 & SMV & - & WS205 & wild soybean & South Korea \\
\hline 38 & FJ640976 & SMV & - & WS209 & wild soybean & South Korea \\
\hline 39 & FJ640977 & SMV & G1 & - & Glycine max & South Korea \\
\hline 40 & FJ640978 & SMV & G3 & - & Glycine max & South Korea \\
\hline 41 & FJ640979 & SMV & G4 & - & Glycine max & South Korea \\
\hline 42 & FJ640980 & SMV & G6 & - & Glycine max & South Korea \\
\hline 43 & FJ640981 & SMV & G6H & - & Glycine max & South Korea \\
\hline 44 & FJ640982 & SMV & G7A & - & Glycine max & South Korea \\
\hline 45 & FJ807700 & SMV & G7H & - & Glycine max & South Korea \\
\hline 46 & FJ807701 & SMV & $\mathrm{G} 5 \mathrm{H}$ & - & Glycine max & South Korea \\
\hline 47 & GU015011 & SMV & - & 413 & $\begin{array}{l}\text { Glycine max (soy- } \\
\text { bean) }\end{array}$ & USA: Illinois \\
\hline 48 & HM590054 & SMV & - & Sc6 & soybean & China \\
\hline 49 & HM590055 & SMV & - & Apr-69 & soybean & China \\
\hline 50 & HQ166265 & SMV & - & NP-C-L & $\begin{array}{l}\text { Glycine max cv. Wil- } \\
\text { liams } 82\end{array}$ & Canada \\
\hline 51 & HQ166266 & SMV & - & NP-L & $\begin{array}{l}\text { Glycine max cv. Wil- } \\
\text { liams } 82\end{array}$ & Canada \\
\hline 52 & HQ396725 & SMV & $4547 / \mathrm{CHN} / 2004$ & - & soybean & China \\
\hline 53 & HQ845735 & SMV & TNP & - & $\begin{array}{l}\text { soybean; genotype } \\
\text { V94-5152 (Rsv4) }\end{array}$ & USA \\
\hline 54 & HQ845736 & SMV & $\mathrm{KY}$ & - & $\begin{array}{l}\text { soybean; genotype } \\
\text { V94-5152 (Rsv4) }\end{array}$ & USA \\
\hline 55 & JF833013 & SMV & - & SC3 & Glycine max & China \\
\hline 56 & JF833014 & SMV & - & Feb-02 & Glycine max & China \\
\hline 57 & JF833015 & SMV & - & Jan-67 & Glycine $\max$ & China \\
\hline 58 & JN416770 & SMV & - & Rsv4-RB3 & Glycine max & Canada \\
\hline 59 & KC845321 & SMV & - & SX & soybean & China \\
\hline 60 & KF135488 & SMV & - & Ar13 & soybean & Iran \\
\hline 61 & KF135489 & SMV & - & Ar33 & soybean & Iran \\
\hline 62 & KF135490 & SMV & - & Lo3 & soybean & Iran \\
\hline 63 & KF135491 & SMV & - & Gol1 & soybean & Iran \\
\hline 64 & KF297335 & SMV & - & Ar33 & soybean & Iran \\
\hline 65 & KM979229 & SMV & - & India & soybean & India \\
\hline 66 & KP710861 & SMV & - & BYX006 & Glycine max & China: Nanchang city \\
\hline 67 & KP710862 & SMV & - & HGT005 & Glycine max & China: Nanchang city \\
\hline 68 & KP710863 & SMV & - & HGT008 & Glycine max & China: Nanchang city \\
\hline 69 & KP710864 & SMV & - & HGT009 & Glycine max & China: Nanchang city \\
\hline 70 & KP710865 & SMV & - & LJZ002 & Glycine max & China: Nanchang city \\
\hline 71 & KP710866 & SMV & - & LJZ010 & Glycine max & China: Nanchang city \\
\hline 72 & KP710867 & SMV & - & SC6-N & Glycine max & China \\
\hline 73 & KP710868 & SMV & - & SC7-N & Glycine max & China \\
\hline 74 & KP710869 & SMV & - & NE-N1 & Glycine max & China: Northeast area \\
\hline 75 & KP710870 & SMV & - & SX-Z & Glycine max & China: Yangling city \\
\hline 76 & KP710871 & SMV & - & XFQ001 & Glycine max & China: Harbin city \\
\hline 77 & KP710872 & SMV & - & XFQ005 & Glycine max & China: Harbin city \\
\hline 78 & KP710873 & SMV & - & XFQ008 & Glycine max & China: Harbin city \\
\hline 79 & KP710874 & SMV & - & XFQ010 & Glycine max & China: Harbin city \\
\hline 80 & KP710875 & SMV & - & XFQ012 & Glycine max & China: Harbin city \\
\hline 81 & KP710876 & SMV & - & XFQ014 & Glycine max & China: Harbin city \\
\hline 82 & KP710877 & SMV & - & XFQ018 & Glycine max & China: Harbin city \\
\hline
\end{tabular}


B. List of the 87 viruses of the "SMV-N" cluster (continued)

\begin{tabular}{llllll}
\hline No. & Acc. No. & Species & Strain & Isolate & Host \\
\hline 83 & KP710878 & SMV & - & XFQ020 & Glycine max \\
84 & KR024718 & SMV & - & JAAS-6 & Soybean \\
85 & KR065437 & SMV & - & HB-RS & Glycine max \\
86 & KT285170 & SMV & - & Jan-78 & Glycine max \\
87 & S42280 & SMV & G2 & - & - \\
\hline
\end{tabular}

C. List of the 3 viruses of the "CLLV-m19" cluster

\begin{tabular}{lllllll}
\hline No. & Acc. No. & Species & Strain & Isolate & Host & Country \\
\hline 1 & NC_021196 & CLLV & m19 & - & Calla lily \\
2 & EF105298 & CLLV & BM19 & - & Calla lily \\
3 & EF105299 & CLLV & E49 & - & Calla lily & Taiwan \\
\hline
\end{tabular}

D. List of the 81 viruses of the "WMV-Fr" cluster

\begin{tabular}{|c|c|c|c|c|c|c|}
\hline No. & Acc. No. & Species & Strain & Isolate & Host & Country \\
\hline 1 & AB218280 & WMV & WMV-Pk & - & - & Pakistan \\
\hline 2 & AB369278 & WMV & Watermelon & - & - & South Korea \\
\hline 3 & NC_006262 & WMV & WMV-Fr & - & - & - \\
\hline 4 & DQ399708 & WMV & WMV-CHN & - & watermelon & China \\
\hline 5 & EU660578 & WMV & - & FMF00-LL2 & - & France \\
\hline 6 & EU660579 & WMV & - & TURK91 & - & Turkey \\
\hline 7 & EU660580 & WMV & - & CHI87-620 & - & Chile \\
\hline 8 & EU660581 & WMV & - & FMF00-LL1 & - & France \\
\hline 9 & EU660582 & WMV & - & CHI02-481 & - & Chile \\
\hline 10 & EU660583 & WMV & - & FMF03-141 & - & France \\
\hline 11 & EU660584 & WMV & - & IR02-54 & - & Iran \\
\hline 12 & EU660585 & WMV & - & $\mathrm{C} 05-270$ & - & France \\
\hline 13 & EU660586 & WMV & - & FBR04-37 & - & France \\
\hline 14 & EU660587 & WMV & - & C06-188 & - & France \\
\hline 15 & EU660588 & WMV & - & C06-666 & - & France \\
\hline 16 & EU660589 & WMV & - & C05-337 & - & France \\
\hline 17 & EU660590 & WMV & - & ITA00-G & - & Italy \\
\hline 18 & FJ823122 & WMV & - & Lecce & Citrullus lanatus & Italy: Lecce \\
\hline 19 & HQ384216 & WMV & Dendrobium & - & $\begin{array}{l}\text { Dendrobium anos- } \\
\text { mum (orchid) }\end{array}$ & USA \\
\hline 20 & JF273458 & WMV & - & $\mathrm{C} 05-463$ & zucchini & France: Garons, Gard \\
\hline 21 & JF273459 & WMV & - & $\mathrm{C} 05-464$ & zucchini & France: Garons, Gard \\
\hline 22 & JF273460 & WMV & - & $\mathrm{C} 05-465$ & zucchini & France: Garons, Gard \\
\hline 23 & JF273461 & WMV & - & C07-349 & melon & $\begin{array}{l}\text { France: Saint Chaptes, } \\
\text { Gard }\end{array}$ \\
\hline 24 & JF273462 & WMV & - & C06-526 & melon & France: Bourdic, Gard \\
\hline 25 & JF273463 & WMV & - & C06-257 & melon & $\begin{array}{l}\text { France: Saint Chaptes, } \\
\text { Gard }\end{array}$ \\
\hline 26 & JF273464 & WMV & - & C07-014 & melon & $\begin{array}{l}\text { France: Aubignan, } \\
\text { Vaucluse }\end{array}$ \\
\hline 27 & JF273465 & WMV & - & A08-160 & zucchini & $\begin{array}{l}\text { France: Aubignan, } \\
\text { Vaucluse }\end{array}$ \\
\hline 28 & JF273466 & WMV & - & A08-170 & zucchini & $\begin{array}{l}\text { France: Aubignan, } \\
\text { Vaucluse }\end{array}$ \\
\hline
\end{tabular}


D. List of the 81 viruses of the "WMV-Fr" cluster (continued)

\begin{tabular}{|c|c|c|c|c|c|c|}
\hline No. & Acc. No. & Species & Strain & Isolate & Host & Country \\
\hline 29 & JF273467 & WMV & - & Cg09-640 & zucchini & $\begin{array}{l}\text { France: Montfavet, } \\
\text { Vaucluse }\end{array}$ \\
\hline 30 & JF273468 & WMV & - & $\mathrm{C} 07-284$ & zucchini & $\begin{array}{l}\text { France: La Tour } \\
\text { d'Aigues, Vaucluse }\end{array}$ \\
\hline 31 & JF273469 & WMV & - & C04-106 & melon & $\begin{array}{l}\text { France: Maillane, } \\
\text { Bouches-du-Rhone }\end{array}$ \\
\hline 32 & JX079685 & WMV & WMV-ShanXi & - & watermelon & China \\
\hline 33 & KC292915 & WMV & - & VE10-099 & Cucumis anguria L. & Venezuela \\
\hline 34 & KM597070 & WMV & - & RKG & watermelon & India \\
\hline 35 & KM597071 & WMV & - & RKG2 & watermelon & India \\
\hline 36 & KP100058 & WMV & - & SangJu6-1 & Panax ginseng & South Korea \\
\hline 37 & KP164988 & WMV & - & WMV 1 SDE FF & - & Argentina \\
\hline 38 & KT992068 & WMV & - & Bonghwa7-2_2014 & $\begin{array}{l}\text { Panax ginseng C.A. } \\
\text { Meyer }\end{array}$ & South Korea \\
\hline 39 & KT992069 & WMV & - & Buan2_2014 & Citrullus vulgaris & South Korea \\
\hline 40 & KT992070 & WMV & - & Buan4-1_2013 & $\begin{array}{l}\text { Panax ginseng C.A. } \\
\text { Meyer }\end{array}$ & South Korea \\
\hline 41 & KT992071 & WMV & - & Cheongsong5_2013 & $\begin{array}{l}\text { Panax ginseng C.A. } \\
\text { Meyer }\end{array}$ & South Korea \\
\hline 42 & KT992072 & WMV & - & Cungju10-1_2014 & $\begin{array}{l}\text { Panax ginseng C.A. } \\
\text { Meyer }\end{array}$ & South Korea \\
\hline 43 & KT992073 & WMV & - & Eumseong1-5_2014 & $\begin{array}{l}\text { Panax ginseng C.A. } \\
\text { Meyer }\end{array}$ & South Korea \\
\hline 44 & KT992074 & WMV & - & Eumseong2-10_2014 & $\begin{array}{l}\text { Panax ginseng C.A. } \\
\text { Meyer }\end{array}$ & South Korea \\
\hline 45 & KT992075 & WMV & - & Hongseong1_2013 & $\begin{array}{l}\text { Panax ginseng C.A. } \\
\text { Meyer }\end{array}$ & South Korea \\
\hline 46 & KT992076 & WMV & - & Naju2-1_2013 & $\begin{array}{l}\text { Panax ginseng C.A. } \\
\text { Meyer }\end{array}$ & South Korea \\
\hline 47 & KT992077 & WMV & - & Naju2-2_2013 & $\begin{array}{l}\text { Panax ginseng C.A. } \\
\text { Meyer }\end{array}$ & South Korea \\
\hline 48 & KT992078 & WMV & - & Sangju2_2014 & $\begin{array}{l}\text { Panax ginseng C.A. } \\
\text { Meyer }\end{array}$ & South Korea \\
\hline 49 & KT992079 & WMV & - & Sangju3_2013 & $\begin{array}{l}\text { Panax ginseng C.A. } \\
\text { Meyer }\end{array}$ & South Korea \\
\hline 50 & KT992080 & WMV & - & Uiseong3-5(sq)_2013 & Cucurbita pepo & South Korea \\
\hline 51 & KT992081 & WMV & - & Yeongam2_2013 & $\begin{array}{l}\text { Panax ginseng C.A. } \\
\text { Meyer }\end{array}$ & South Korea \\
\hline 52 & KT992082 & WMV & - & Yeongam4-2_2014 & $\begin{array}{l}\text { Panax ginseng C.A. } \\
\text { Meyer }\end{array}$ & South Korea \\
\hline 53 & KT992083 & WMV & - & Yeongju1-2_2013 & $\begin{array}{l}\text { Panax ginseng C.A. } \\
\text { Meyer }\end{array}$ & South Korea \\
\hline 54 & KT992084 & WMV & - & Yeongju2-1_2014 & $\begin{array}{l}\text { Panax ginseng C.A. } \\
\text { Meyer }\end{array}$ & South Korea \\
\hline 55 & KT992085 & WMV & - & Yeongju2-3_2013 & $\begin{array}{l}\text { Panax ginseng C.A. } \\
\text { Meyer }\end{array}$ & South Korea \\
\hline 56 & KT992086 & WMV & - & Yeongju6-1_2013 & $\begin{array}{l}\text { Panax ginseng C.A. } \\
\text { Meyer }\end{array}$ & South Korea \\
\hline 57 & KT992087 & WMV & - & Yeongju7-1_2014 & $\begin{array}{l}\text { Panax ginseng C.A. } \\
\text { Meyer }\end{array}$ & South Korea \\
\hline 58 & KT992088 & WMV & - & Yeongju7-2_2013 & $\begin{array}{l}\text { Panax ginseng C.A. } \\
\text { Meyer }\end{array}$ & South Korea \\
\hline 59 & KT992089 & WMV & - & Yeongju7-2(cm)_2013 & Malva verticillata & South Korea \\
\hline 60 & KT992090 & WMV & - & Yeongju7-3_2013 & $\begin{array}{l}\text { Panax ginseng C.A. } \\
\text { Meyer }\end{array}$ & South Korea \\
\hline
\end{tabular}


D. List of the 81 viruses of the "WMV-Fr" cluster (continued)

\begin{tabular}{|c|c|c|c|c|c|c|}
\hline No. & Acc. No. & Species & Strain & Isolate & Host & Country \\
\hline 61 & KT992091 & WMV & - & Yeongju7-5(sq)_2013 & Cucurbita pepo & South Korea \\
\hline 62 & KT992092 & WMV & - & Yeongju9_2014 & $\begin{array}{l}\text { Panax ginseng C.A. } \\
\text { Meyer }\end{array}$ & South Korea \\
\hline 63 & KT992093 & WMV & - & Yeongyang8-1_2013 & $\begin{array}{l}\text { Panax ginseng C.A. } \\
\text { Meyer }\end{array}$ & South Korea \\
\hline 64 & KU240094 & WMV & - & Buan2-1_2012 & - & South Korea \\
\hline 65 & KU240095 & WMV & - & Gimcheon1_2012 & - & South Korea \\
\hline 66 & KU240096 & WMV & - & Gochang2_2012 & - & South Korea \\
\hline 67 & KU240097 & WMV & - & Gumi4_2012 & - & South Korea \\
\hline 68 & KU240098 & WMV & - & Jeongeup1-1_2012 & - & South Korea \\
\hline 69 & KU240099 & WMV & - & Jeongeup_3-1_2012 & - & South Korea \\
\hline 70 & KU240100 & WMV & - & Naju3-1_2012 & - & South Korea \\
\hline 71 & KU240101 & WMV & - & Pungjeonglee1_2012 & - & South Korea \\
\hline 72 & KU240102 & WMV & - & Pungjeonglee3_2012 & - & South Korea \\
\hline 73 & KU240103 & WMV & - & Sangju2_2012 & - & South Korea \\
\hline 74 & KU240104 & WMV & - & Sangju6-1_2012 & - & South Korea \\
\hline 75 & KU240105 & WMV & - & Sangju6_2012 & - & South Korea \\
\hline 76 & KU240106 & WMV & - & Sangju7-1_2012 & - & South Korea \\
\hline 77 & KU240107 & WMV & - & Yeongam2-1_2012 & - & South Korea \\
\hline 78 & KU240108 & WMV & - & Yeongju5_2012 & - & South Korea \\
\hline 79 & KU240109 & WMV & - & Yeongju9_2012 & - & South Korea \\
\hline 80 & KU240110 & WMV & - & Yeongyang3_2012 & - & South Korea \\
\hline 81 & KU246036 & WMV & - & TX29 & watermelon & USA \\
\hline
\end{tabular}

